\title{
FUNCTION SPACES FOR CONDITIONALLY POSITIVE DEFINITE OPERATOR-VALUED KERNELS
}

\author{
GEORG BERSCHNEIDER, WOLFGANG ZU CASTELL, AND STEFAN J. SCHRÖDL
}

\begin{abstract}
The correspondence between reproducing kernel Hilbert spaces and positive definite kernels is well understood since Aronszajn's work of the 1940s. The analog relation is less clear for conditionally positive definite kernels. The latter are widely used in approximation methods for scattered data, geostatistics, and machine learning. We consider this relation and provide two ways to construct a reproducing kernel Pontryagin space for operator-valued kernels.
\end{abstract}

\section{INTRODUCTION}

Approximation with linear combinations of kernels is a widely used method in approximation theory [18, spatial statistics (kriging) [6] and machine learning [15]. The approach is appealing since there are almost no restrictions on the geometry of the measurements (scattered data), and the kernel itself can be understood as a way to incorporate a priori information concerning similarity within the data. These approaches heavily rely on an underlying reproducing kernel space. For example, the idea of the "kernel trick" in machine learning (cf. [15]) is to map the data into the so-called feature space where the actual algorithm is carried out. In approximation theory several authors studied the spaces resulting from an approximation process based on translates of positive definite, radial functions on $\mathbb{R}^{d}$ (cf. [11, 14]).

Let us briefly recall the definition of conditionally positive definite kernels (cf. 1, 12, 16]). Therefore, let $X$ be an arbitrary non-empty set and $(V,\langle\cdot, \cdot\rangle)$ a Pontryagin space over the complex field $\mathbb{C}$ (see Section 2 below for a definition). By $B(V)$ we denote the set of bounded linear operators on $V$. For $A \in B(V), A^{*}$ will be its adjoint operator.

We call a function $\Phi: X \times X \rightarrow B(V)$ conditionally positive definite (operatorvalued) kernel of order $\kappa \in \mathbb{N}_{0}$, if it is hermitian, i.e.,

$$
\Phi(y, x)=\Phi(x, y)^{*} \quad \forall x, y \in X
$$

and there exists a $\kappa$-dimensional space $\mathcal{U}$ of functions from $X$ to $V$ such that

$$
\sum_{i=1}^{n} \sum_{j=1}^{n}\left\langle\Phi\left(x_{i}, x_{j}\right) v_{j}, v_{i}\right\rangle \geq 0
$$

Received by the editor April 8, 2010 and, in revised form, March 8, 2011.

2010 Mathematics Subject Classification. Primary 46C20, 41A63, 47A56; Secondary 41A05, $62 \mathrm{M} 30,62 \mathrm{H} 30$.

(C)2011 American Mathematical Society Reverts to public domain 28 years from publication 
holds for every choice of finitely many points $x_{1}, \ldots, x_{n} \in X$ and vectors $v_{1}, \ldots, v_{n} \in$ $V$ satisfying

$$
\sum_{i=1}^{n}\left\langle u\left(x_{i}\right), v_{i}\right\rangle=0 \quad \forall u \in \mathcal{U} .
$$

If the space $\mathcal{U}$ is fixed, we will call $\Phi$ conditionally positive definite with respect to $\mathcal{U}$. A positive definite kernel is conditionally positive definite with respect to the trivial subspace. Note that the choice of the order to be equal to the dimension of the space $\mathcal{U}$ differs from the analog notion, when dealing with spaces of polynomials.

Conditionally positive definite operator-valued kernels have found their way into applications, too (cf. 2, 7, 9, 10]). In spatial statistics such methods are known as cokriging and multivariate spatial statistics [6], while in machine learning the notion of multi-task learning 8 is used.

We want to study the underlying function spaces associated with conditionally positive definite kernels. These spaces turn out to be reproducing kernel Pontryagin spaces [13, 17. For some examples of radial basis functions these spaces can be identified with Sobolev spaces or Beppo-Levi spaces (cf. [2, 7, 9, 10, 11]). Note that all those examples deal with translation invariant kernels and thus require some additional structure on the space $X$.

For a first construction in Section 3 we follow the approach given in 3 . In the work mentioned, it has been shown that associated with a scalar-valued, conditionally positive definite kernel $\Phi$ of order $\kappa$ there is a reproducing kernel Pontryagin space of index $\gamma \leq \kappa$ such that $\Phi$ is its reproducing kernel. Note that such a statement does not make use of the explicit nature of the space $\mathcal{U}$.

In many applications the space $\mathcal{U}$ has some interpretation. For example, within the context of radial basis function interpolation one commonly chooses $\mathcal{U}$ to be the space of polynomials on $\mathbb{R}^{d}$ of some finite degree. Similarly, in statistical applications $\mathcal{U}$ might model a polynomial trend within an otherwise stationary phenomenon. In learning theory $\mathcal{U}$ would model the parametric component of a semiparametric learning scheme. Therefore, one would like to choose a Pontryagin space with negative subspace $\mathcal{U}$. We will give such a construction in the second approach in Section 4 . Naturally, one can then no longer expect $\Phi$ to be the reproducing kernel.

Before presenting the two constructions we briefly review some facts on reproducing kernel spaces in the following section. We conclude with discussing some aspects related to applications and stating some examples.

\section{Preliminaries}

For the constructions mentioned we need to calculate projections onto the finitedimensional negative subspace. Considering given data as evaluations of an unknown function, it seems natural to use so-called interpolating projections. These rely on the existence of a set of finitely many points which determine the negative subspace uniquely.

We say that a set $\Xi \subseteq X$ is a $\mathcal{U}$-unisolvent set of points, if every element $u \in \mathcal{U}$ vanishing on $\Xi$ is identically zero. In the scalar case the existence of finite $\mathcal{U}$-unisolvent sets follows from $\mathcal{U}$ being finite-dimensional and Auerbach's Theorem (cf. [5. Chapter 31, Lemma 2]). The proof actually shows the existence of a linearly 
independent set in the dual space $\mathcal{U}^{\prime}$. Since this argument cannot be applied directly in the case of vector-valued functions we give a direct proof.

Lemma 2.1. Let $V$ be a vector space over the field $\mathbb{C}$ and $\mathcal{U}$ a finite-dimensional space of $V$-valued functions on $X$. Then there exists a $\mathcal{U}$-unisolvent set of points $\Xi \subset X$ with $|\Xi|<\infty$.

Proof. For a finite set $X$, we can choose $\Xi=X$. Therefore, assume $|X|=\infty$. We proceed by induction on the dimension $n$ of $\mathcal{U}$. For $n=1$ there is a $u_{0}: X \rightarrow V$ such that $\mathcal{U}=\operatorname{span}\left\{u_{0}\right\}$ and $\xi \in X$ such that $u_{0}(\xi) \neq 0$. Now suppose $\operatorname{dim} \mathcal{U}=n+1$ and write

$$
\mathcal{U}=\mathcal{W} \oplus \operatorname{span}\left\{u_{0}\right\}
$$

where $u_{0} \in \mathcal{U}, u_{0} \neq 0$. Since $\operatorname{dim} \mathcal{W}=n$ the induction hypothesis yields the existence of a finite $\mathcal{W}$-unisolvent set $\Xi_{n} \subset X$. Suppose for every $x \in X \backslash \Xi_{n}$ there is a $u_{x} \in \mathcal{U} \backslash\{0\}$ such that $u_{x}(\xi)=0$ for all $\xi \in \Xi_{n} \cup\{x\}$.

Choose $x_{1}, x_{2} \in X \backslash \Xi_{n}, x_{1} \neq x_{2}$ and let $u_{1}=u_{x_{1}}, u_{2}=u_{x_{2}}$ be as described. Then

$$
u_{i}=u_{i}^{\prime}+\alpha_{i} u_{0}, \quad i=1,2,
$$

where $u_{i}^{\prime} \in \mathcal{W}$ and $\alpha_{i} \neq 0$, because otherwise $u_{i}=0$ by induction hypothesis. Thus,

$$
u_{0}(\xi)=-\frac{1}{\alpha_{i}} u_{i}^{\prime}(\xi) \quad \forall \xi \in \Xi_{n} \cup\left\{x_{i}\right\} .
$$

Setting $v_{i}=-\frac{1}{\alpha_{i}} u_{i}^{\prime} \in \mathcal{W}$, we obtain that $v_{1}(\xi)=u_{0}(\xi)=v_{2}(\xi)$ for all $\xi \in \Xi_{n}$, hence $v_{1}=v_{2}=v$. Since $x_{1}, x_{2}$ were arbitrary it follows from equation (2.1) that $u_{0}=v$. Therefore, $u_{0} \in \mathcal{W}$, which is a contradiction.

In contrast to the scalar-valued case the minimal size of a unisolvent set can be smaller than the actual dimension of the space. As an example consider the space of functions $p: \mathbb{C} \rightarrow \mathbb{C}^{m}$, where every coordinate function is a polynomial of degree at most $n$. Then $\operatorname{dim} \mathcal{U}=(n+1) m$, but $(n+1)$ points suffice to determine $p \in \mathcal{U}$ uniquely.

Reproducing kernel Pontryagin spaces. Let us turn our attention to Pontryagin spaces and collect some basic facts. For further details the reader is referred to the monographs [1, 4, 13.

Given a Hilbert space $H$ and replacing the inner product with its negative defines the corresponding antispace. A Pontryagin space $\Pi$ then is a space which can be written as a direct sum $\Pi=N \oplus N^{\perp}$, where $\left(N^{\perp},\langle\cdot, \cdot\rangle_{N^{\perp}}\right)$ is a Hilbert space and $\left(N,\langle\cdot, \cdot\rangle_{N}\right)$ is the antispace of a finite-dimensional Hilbert space. The space $\Pi$ is endowed with the indefinite inner product

$$
(v, w)=\left\langle v_{+}, w_{+}\right\rangle_{N^{\perp}}+\left\langle v_{-}, w_{-}\right\rangle_{N}, \quad v_{+}, w_{+} \in N^{\perp}, v_{-}, w_{-} \in N .
$$

Such a decomposition is called fundamental decomposition. The space $N$ is called negative subspace of $\Pi$, since from the definition we immediately get that $(v, v)<0$ for all $v \in N \backslash\{0\}$. Note that $N$ is maximal with respect to this property, i.e., not included in any larger negative subspace.

While a fundamental decomposition of a Pontryagin space is not unique, the dimension of its maximal negative subspace $N$ is and is therefore referred to as the index of the Pontryagin space. Given a fundamental decomposition of $\Pi$ the inner product

$$
[v, w]=\left(v_{+}, w_{+}\right)-\left(v_{-}, w_{-}\right), \quad v_{+}, w_{+} \in N^{\perp}, v_{-}, w_{-} \in N,
$$


turns $\Pi$ into a Hilbert space. All norms induced in such a way can be shown to be equivalent (cf. [4, (IX.2)]). It is the topology induced by such a norm which we will use on the space $\Pi$.

As a consequence of a theorem by Pontryagin one can show the following statements.

Lemma 2.2 ([1, Lemma 1.1.1]). Let $\Pi$ be a Pontryagin space of index $\gamma \in \mathbb{N}_{0}$.

(i) The Gram matrix $\mathrm{G}=\left[\left(v_{i}, v_{j}\right)\right]_{i, j=1}^{n}$ of any number of finitely many vectors $v_{1}, \ldots, v_{n} \in \Pi$ can have at most $\gamma$ negative eigenvalues (counted with multiplicity).

(ii) If $T \subseteq \Pi$ is a total set, i.e., its span is dense in $\Pi$, then $T$ contains a finite subset whose Gram matrix has exactly $\gamma$ negative eigenvalues (counted with multiplicity).

Definition 2.3. Let $V$ be a Pontryagin space and $\mathcal{H}$ be a Pontryagin space of functions $f: X \rightarrow V$, where $X$ is some non-empty set. A kernel $\Phi$ mapping $X \times X$ into the space $B(V)$ is called reproducing kernel of $\mathcal{H}$ if

(i) $\Phi(\cdot, x) v$ belongs to $\mathcal{H}$ for every $x \in X$ and $v \in V$, and

(ii) for every $x \in X, f \in \mathcal{H}$, and $v \in V$,

$$
(f, \Phi(\cdot, x) v)=\langle f(x), v\rangle .
$$

If such a kernel exists, $\mathcal{H}$ is called reproducing kernel Pontryagin space.

The Pontryagin space analog of Riesz' representation theorem (cf. [13, (B.1)]) shows that the reproducing property is tied up with the existence of continuous evaluation mappings

$$
E_{x}: \mathcal{H} \rightarrow V, f \mapsto f(x), \quad x \in X
$$

We then have the following characterization (cf. [1, Theorem 1.1.2] and [12]).

Theorem 2.4. Let $\mathcal{H}$ be a Pontryagin space of functions $f: X \rightarrow V$, where $V$ is a Pontryagin space and $X$ is non-empty. Then $\mathcal{H}$ is a reproducing kernel Pontryagin space if and only if for all $x \in X$ the evaluation mapping $E_{x}: \mathcal{H} \rightarrow V$ is continuous. If the latter is the case and $\Phi: X \times X \rightarrow B(V)$ is a reproducing kernel, $\Phi$ is unique and satisfies the following properties:

(i) $\Phi(x, y)=E_{x} E_{y}^{*}$ for all $x, y \in X$.

(ii) $\Phi(x, y)=\Phi(y, x)^{*}$ for all $x, y \in X$.

(iii) The set

$$
\{\Phi(\cdot, x) v: x \in X, v \in V\}
$$

forms a total subset of $\mathcal{H}$.

Let $\gamma$ be the index of the reproducing kernel Pontryagin space $\mathcal{H}$. From Lemma 2.2 and Theorem 2.4 it follows that for any finite set of points $\left\{x_{1}, \ldots, x_{n}\right\} \subseteq$ $X$ and vectors $\left\{v_{1}, \ldots, v_{n}\right\} \subseteq V$ the Gram matrix $\left[\left\langle\Phi\left(x_{i}, x_{j}\right) v_{j}, v_{i}\right\rangle\right]_{i, j=1}^{n}$ has at most $\gamma$ negative eigenvalues. Since $\{\Phi(\cdot, x) v: x \in X, v \in V\}$ is total in $\mathcal{H}$, it therefore contains at least one finite set $\left\{\Phi\left(\cdot, x_{1}\right) v_{1}, \ldots, \Phi\left(\cdot, x_{n}\right) v_{n}\right\}$ such that the corresponding Gram matrix has exactly $\gamma$ negative eigenvalues. Therefore, $\Phi$ is said to have $\gamma$ negative squares.

Sorjonen [17] showed that there is a one-to-one correspondence between reproducing kernel Pontryagin spaces and kernels with finitely many negative squares. 
Theorem 2.5 (Sorjonen, [17]). Suppose $\gamma \in \mathbb{N}_{0}$ and let $V$ be a Pontryagin space. If $\mathcal{H}$ is a Pontryagin space of index $\gamma$ with reproducing kernel $\Phi$, then $\Phi$ has $\gamma$ negative squares. Conversely, for every hermitian kernel $\Phi: X \times X \rightarrow B(V)$ with $\gamma$ negative squares there exists a Pontryagin space $\mathcal{H}$ of index $\gamma$ with reproducing kernel $\Phi$.

\section{Direct COnstruction of a FunCtion SPACE}

Let us now consider a conditionally positive definite operator-valued kernel $\Phi$ and describe the construction for a corresponding reproducing kernel Pontryagin space using the kernel directly. If $\Phi$ were positive definite and scalar-valued, the general construction of the associated reproducing kernel Hilbert space is to start with considering the space $\operatorname{span}\{\Phi(\cdot, x): x \in X\}$ and define a sesquilinear form on this space derived from the kernel. Due to the property of being positive definite this form can easily be seen to be a proper inner product. Then taking the completion with respect to this inner product leads to the reproducing kernel Hilbert space with reproducing kernel $\Phi$. Within the context of representation theory this method has been used by Gel'fand and Naimark and later was extended by Segal to construct representation spaces for certain normed algebras. It is for this reason that the construction is referred to as GNS-construction within harmonic analysis.

Basically, the idea to construct a reproducing kernel Pontryagin space associated with a given conditionally positive definite kernel uses the same construction. But since such a kernel does define an indefinite inner product only, one has more freedom, thus leading to different alternatives.

One way is to use the correspondence between conditionally positive definite kernels and kernels with finitely many negative squares. This is the approach followed in [3] for the scalar-valued case. Doing the GNS-construction with a conditionally positive definite kernel $\Phi$ of order $\kappa \in \mathbb{N}_{0}$ leads to a pre-Pontryagin space of index $\gamma$, where $0 \leq \gamma \leq \kappa$. Using a result of Sorjonen [17, Satz 3.2] allows us to take the completion and thereby derive a Pontryagin space with reproducing kernel $\Phi$.

The construction for the operator-valued case follows along the lines of the scalarvalued case. Therefore, we restrict ourselves to stating the essential steps of the construction.

To be precise, assume $(V,\langle\cdot, \cdot\rangle)$ to be a Pontryagin space, $X \neq \emptyset$, and $\Phi$ : $X \times X \rightarrow B(V)$ to be a conditionally positive definite kernel of order $\kappa \in \mathbb{N}_{0}$. Being an operator, $\Phi_{x}=\Phi(\cdot, x)$ is not an element of the reproducing kernel space. Thus, we consider as a starting point the linear space

$$
\mathcal{T}=\operatorname{span}\left\{\Phi_{x} v: x \in X, v \in V\right\} .
$$

For any two elements $f, g \in \mathcal{T}$ we can find points $x_{1}, \ldots, x_{n} \in X$ and vectors $v_{1}, \ldots, v_{n}, w_{1}, \ldots, w_{n} \in V$ such that

$$
f=\sum_{i=1}^{n} \Phi_{x_{i}} v_{i} \text { and } g=\sum_{i=1}^{n} \Phi_{x_{i}} w_{i}
$$

Since

$$
\sum_{i=1}^{n}\left\langle f\left(x_{i}\right), w_{i}\right\rangle=\sum_{i, j=1}^{n}\left\langle\Phi\left(x_{i}, x_{j}\right) v_{j}, w_{i}\right\rangle=\sum_{j=1}^{n}\left\langle v_{j}, g\left(x_{j}\right)\right\rangle
$$


the form

$$
(f, g)=\sum_{i, j=1}^{n}\left\langle\Phi\left(x_{i}, x_{j}\right) v_{j}, w_{i}\right\rangle
$$

defines a hermitian sesquilinear form on $\mathcal{T}$. It is easy to check that $(\cdot, \cdot)$ satisfies

$$
\left(f, \Phi_{x} v\right)=\langle f(x), v\rangle
$$

for all $f \in \mathcal{T}, x \in X$, and $v \in V$. This also guarantees non-degeneracy. To see this, let $f_{0} \in \mathcal{T}$ with $\left(f_{0}, f\right)=0$ for all $f \in \mathcal{T}$. Choosing $f=\Phi_{x} v$ leads to

$$
0=\left(f_{0}, \Phi_{x} v\right)=\left\langle f_{0}(x), v\right\rangle
$$

for all $v \in V$. Non-degeneracy of $V$ implies $f_{0}=0$.

The results so far sum up to the following proposition.

Proposition 3.1. Let $\Phi: X \times X \rightarrow B(V)$ be a conditionally positive definite kernel of order $\kappa$. The space $\mathcal{T}$ endowed with $(\cdot, \cdot)$ is a pre-Pontryagin space of index $\gamma$, $\gamma \leq \kappa$, having $\Phi$ as its reproducing kernel.

Proof. It remains to show that any maximal negative subspace of $\mathcal{T}$ is of dimension $\gamma, \gamma \leq \kappa$. Therefore, choose $n \geq \kappa$ points $x_{1}, \ldots, x_{n} \in X$ and vectors $v_{1}, \ldots, v_{n} \in V$ and consider the Gram matrix

$$
\mathrm{G}=\left[\left(\Phi_{x_{j}} v_{j}, \Phi_{x_{i}} v_{i}\right)\right]_{i, j=1}^{n} .
$$

From the defining equations (1.1) and (1.2) for conditional positive definiteness we obtain that

$$
\mathbf{a}^{*} \mathrm{Ga} \geq 0
$$

for all $\mathbf{a} \in \mathbb{C}^{n}$ satisfying

$$
\sum_{i=1}^{n} \overline{a_{i}}\left\langle u\left(x_{i}\right), v_{i}\right\rangle=0 \quad \forall u \in \mathcal{U}
$$

or, equivalently,

$$
\mathbf{a} \in\left\{\left[\left\langle u\left(x_{i}\right), v_{i}\right\rangle\right]_{i=1}^{n}: u \in \mathcal{U}\right\}^{\perp}=W^{\perp} \subseteq \mathbb{C}^{n} .
$$

Since $\operatorname{dim} W \leq \operatorname{dim} \mathcal{U}=\kappa, G$ can have at most $\kappa$ negative eigenvalues. Using Lemma 2.2 any negative subspace in $\operatorname{span}\left\{\Phi_{x_{1}} v_{1}, \ldots, \Phi_{x_{n}} v_{n}\right\}$ is of dimension less than the number of negative eigenvalues of the Gram matrix. Let $\gamma$ be the maximal number of negative eigenvalues occurring for arbitrary choices of finitely many points. A negative subspace cannot be infinite dimensional since the existence of a negative subspace of dimension greater than $\kappa$ immediately results in a contradiction.

Theorem 3.2. Let $\Phi: X \times X \rightarrow B(V)$ be a conditionally positive definite kernel of order $\kappa$. Then there exists a Pontryagin space $\mathcal{H}$ of index $\gamma, \gamma \leq \kappa$, such that $\Phi$ is its reproducing kernel and

$$
\operatorname{span}\left\{\Phi_{x} v: x \in X, v \in V\right\}
$$

is dense in $\mathcal{H}$.

Proof. Consider the pre-Pontryagin space $\mathcal{T}$ endowed with $(\cdot, \cdot)$ as in Proposition 3.1. Using Sorjonen's result [17, Satz 3.2] leads to the Pontryagin space completion $\mathcal{H}$ of $\mathcal{T}$. 
As in the positive definite case there is a reproducing kernel Pontryagin space for every conditionally positive definite kernel. The converse result is given in the following theorem. The proof follows along the lines of the scalar-valued case (cf. [3]) and is therefore omitted.

Theorem 3.3. Let $V$ be a Pontryagin space and $\mathcal{H}$ be a Pontryagin space of index $\gamma$ of $V$-valued functions on $X$ with reproducing kernel $\Phi$. Then $\Phi$ is conditionally positive definite of order $\gamma$. In particular, $\Phi$ is conditionally positive definite with respect to any maximal negative subspace of $\mathcal{H}$.

As already pointed out in Section 2, every maximal negative subspace $\mathcal{N}$ of $\mathcal{H}$ defines an inner product $[\cdot, \cdot]_{\mathcal{N}}$, which turns $\mathcal{H}$ into a reproducing kernel Hilbert space. The representation of its reproducing kernel $\Lambda: X \times X \rightarrow B(V)$ in terms of $\Phi$ is given in the next theorem.

Theorem 3.4. Let $\Phi: X \times X \rightarrow B(V)$ be a conditionally positive definite kernel of order $\kappa \in \mathbb{N}_{0}$ and $\mathcal{H}$ the associated reproducing kernel Pontryagin space. Choose a maximal negative subspace $\mathcal{N}$ of $\mathcal{H}$ and an orthonormal basis $\left\{p_{1}, \ldots, p_{\lambda}\right\}$ of $\mathcal{N}$. Then

$$
\Lambda(x, y)=\Phi(x, y)+2 \sum_{i=1}^{\lambda} p_{i}(x) p_{i}(y)^{*}, \quad x, y \in X
$$

is the reproducing kernel of $\mathcal{H}$ corresponding to $[\cdot, \cdot]_{\mathcal{N}}$.

Although the direct construction has the advantage that the kernel $\Phi$ we started with has a direct interpretation as reproducing kernel, the space $\mathcal{U}$ does in general not appear within the geometric decomposition $\mathcal{N} \oplus \mathcal{N}^{\perp}$ of the Pontryagin space $\mathcal{H}$.

Posing further assumptions on the kernel we can identify $\mathcal{U}$ as a subspace of the resulting reproducing kernel Pontryagin space. Towards this end, let us assume that $\Phi$ is conditionally positive definite with respect to $\mathcal{U}$. Let $\mathcal{U}$ be minimal with respect to this property, i.e., $\Phi$ is not conditionally positive definite with respect to any proper subspace of $\mathcal{U}$. Denote by $\mathcal{H}$ the reproducing kernel Pontryagin space associated with $\Phi$ according to Theorem 3.2. Consider $f \in \mathcal{H}$ such that for every finite choice of points $x_{1}, \ldots, x_{n} \in X$ and vectors $v_{1}, \ldots, v_{n} \in V$ with

$$
\sum_{i=1}^{n}\left\langle u\left(x_{i}\right), v_{i}\right\rangle=0 \quad \forall u \in \mathcal{U}
$$

we have that

$$
\sum_{i=1}^{n}\left\langle f\left(x_{i}\right), v_{i}\right\rangle=0
$$

Then $f \in \mathcal{U}$. Indeed, fix points $x_{1}, \ldots, x_{n} \in X$ and let

$$
W=\left\{\mathbf{u}=\left[u\left(x_{i}\right)\right]_{i=1}^{n}: u \in \mathcal{U}\right\} \subseteq V^{n} .
$$

Then $W$ is a finite-dimensional subspace of the Pontryagin space $V^{n}$. Setting $\mathbf{f}=\left[f\left(x_{i}\right)\right]_{i=1}^{n}$ and $\mathbf{v}=\left[v_{i}\right]_{i=1}^{n}$ we have that $\langle\mathbf{u}, \mathbf{v}\rangle=0$ and $\langle\mathbf{f}, \mathbf{v}\rangle=0$. Therefore,

$$
\mathbf{f} \in\left(W^{\perp}\right)^{\perp}=W
$$

i.e., there exists an element $u \in \mathcal{U}$ such that

$$
f\left(x_{i}\right)=u\left(x_{i}\right), \quad 1 \leq i \leq n .
$$


Using the following lemma we then conclude that $f \in \mathcal{U}$.

Lemma 3.5. Let $V$ be an arbitrary vector space over the field $\mathbb{C}$ and $\mathcal{U}$ be a finitedimensional space of $V$-valued functions on $X \neq \emptyset$. Let $f: X \rightarrow V$. If for arbitrary $n \in \mathbb{N}$ and all choices of $n$ points $x_{1}, \ldots, x_{n} \in X$ there exists a function $u \in \mathcal{U}$ depending on $x_{1}, \ldots, x_{n}$ with

$$
f\left(x_{i}\right)=u\left(x_{i}\right), \quad 1 \leq i \leq n,
$$

then $f \in \mathcal{U}$.

Proof. If $X$ is finite, the assertion is trivially satisfied. Thus, suppose that $|X|=\infty$. Since $\mathcal{U}$ is finite-dimensional Lemma 2.1 yields the existence of a finite $\mathcal{U}$-unisolvent set $\Xi \subset X$ of points. Let $u_{\Xi} \in \mathcal{U}$ be chosen according to the assumptions. Fix $x \in X \backslash \Xi$ and let $u_{x} \in \mathcal{U}$ such that $f(\xi)=u_{x}(\xi)$ for all $\xi \in \Xi \cup\{x\}$. Since

$$
u_{x}(\xi)=f(\xi)=u_{\Xi}(\xi) \quad \forall \xi \in \Xi
$$

$u_{\Xi}=u_{x}$ by the unisolvency of $\Xi$. Since $x$ was arbitrary it follows that $f=u_{\Xi}$.

Returning to the problem of identifying $\mathcal{U}$ as a subspace of $\mathcal{H}$ we consider the space

$$
\mathcal{F}=\left\{\sum_{i=1}^{n} \Phi_{x_{i}} v_{i}: \sum_{i=1}^{n}\left\langle u\left(x_{i}\right), v_{i}\right\rangle=0 \forall u \in \mathcal{U}\right\} \subseteq \mathcal{H} .
$$

From the remarks preceding Lemma 3.5 we obtain that $\mathcal{F}^{\perp} \subseteq \mathcal{U}$. Assuming $\mathcal{F}^{\perp}$ to be a proper subspace of $\mathcal{U}$ we can find a basis $\left\{u_{1}, \ldots, u_{m}\right\}$ of $\mathcal{F}^{\perp}$ and $u_{m+1}, \ldots, u_{\kappa} \in \mathcal{U}$ such that $\left\{u_{1}, \ldots, u_{\kappa}\right\}$ forms a basis of $\mathcal{U}$. Choose finitely many points $x_{1}, \ldots, x_{n} \in X$ and vectors $v_{1}, \ldots, v_{n} \in V$ such that

$$
\sum_{i=1}^{n}\left\langle u_{j}\left(x_{i}\right), v_{i}\right\rangle=0, \quad 1 \leq j \leq m
$$

Then

$$
f=\sum_{i=1}^{n} \Phi_{x_{i}} v_{i} \in\left(\mathcal{F}^{\perp}\right)^{\perp}=\overline{\mathcal{F}}
$$

From the defining relations (1.1) and (1.2) of conditional positive definiteness we obtain that $\mathcal{F}$ is a non-negative subspace of $\mathcal{H}$. Thus, it is easy to see that $f \in \overline{\mathcal{F}}$ implies conditional positive definiteness of $\Phi$ with respect to $\mathcal{F}^{\perp}$. This contradicts the assumption of minimality of the space $\mathcal{U}$.

Summarizing, we have the following result.

Theorem 3.6. Let $(V,\langle\cdot, \cdot\rangle)$ be a Pontryagin space and $\Phi: X \times X \rightarrow B(V)$ be conditionally positive definite with respect to a finite-dimensional space $\mathcal{U}$. Assume $\mathcal{U}$ to be minimal with respect to this property. Then $\mathcal{U}$ is contained in the reproducing kernel space $\mathcal{H}$ associated with $\Phi$.

\section{Construction using a projected kernel}

We already noted in the preceding section that the construction of a function space starting from a conditionally positive definite kernel $\Phi$ of index $\kappa$ generally results in a reproducing kernel Pontryagin space $\mathcal{H}$ of index $\gamma \leq \kappa$. In particular, fixing a $\kappa$-dimensional space $\mathcal{U}$ for the kernel $\Phi$ we can in general not expect $\mathcal{U}$ to be a maximal negative subspace of $\mathcal{H}$. In this section we consider a second construction aiming for a reproducing kernel Pontryagin space with maximal negative subspace 
$\mathcal{U}$. A similar construction for scalar-valued kernels may also be found in [5, Chapter 31].

Given an orthonormal basis $\left\{p_{1}, \ldots, p_{\kappa}\right\}$ of $\mathcal{U}$ the reproducing kernel of $\mathcal{U}$ has the form $\sum_{i=1}^{\kappa} p_{i}(x) p_{i}(y)^{*}$ due to $\mathcal{U}$ being finite-dimensional. The idea of the construction is to "subtract" $\mathcal{U}$ from a reproducing kernel Hilbert space obtained via the GNS-construction. In order to make this work we have to ensure that the kernel $\Psi$ used for the GNS-construction does not contain any component in $\mathcal{U}$. The key lies in determining a suitable projection of the given conditionally positive definite kernel $\Phi$ onto $\mathcal{U}$.

Let us restrict our considerations for the moment to the scalar-valued case, i.e., $\mathcal{U}$ consists of complex-valued functions. Choosing a Lagrange or cardinal basis of $\mathcal{U}$ allows to compute projections in terms of point evaluations. To be precise, choose a minimal $\mathcal{U}$-unisolvent set $\Xi=\left\{\xi_{1}, \ldots, \xi_{\kappa}\right\}$ of points. The Lagrange basis of $\mathcal{U}$ with respect to $\Xi$ consists of those functions $u_{i} \in \mathcal{U}$ satisfying

$$
u_{j}\left(\xi_{i}\right)=\delta_{i, j}, \quad 1 \leq i, j \leq \kappa .
$$

With this property, any element $u \in \mathcal{U}$ has a representation

$$
u(x)=\sum_{i=1}^{\kappa} u\left(\xi_{i}\right) u_{i}(x), \quad x \in X .
$$

For $\mathcal{U}$ being a space of polynomials this is the Lagrange interpolation formula (cf. [5, Chapter 2]).

Let us turn back to the general case of $V$-valued functions. In order to compute the desired projection in terms of point evaluations we have to find a vector-analog of the Lagrange basis. The idea is to consider a finite-dimensional space $\widetilde{\mathcal{U}}$ of complex-valued functions on $X$ associated with the given space $\mathcal{U}$ consisting of $V$-valued functions.

Towards this end, let $\widetilde{\mathcal{U}}$ be the space of all functions

$$
\widetilde{u}: X \times V \rightarrow \mathbb{C},(x, v) \mapsto \widetilde{u}(x, v)=\langle u(x), v\rangle,
$$

where $u \in \mathcal{U}$. The spaces $\mathcal{U}$ and $\widetilde{\mathcal{U}}$ are isomorphic and are said to be in tilde correspondence (cf. [12]).

Since $\widetilde{\mathcal{U}}$ is a finite-dimensional space of complex-valued functions on $X \times V$, we can find a minimal $\widetilde{\mathcal{U}}$-unisolvent set $\widetilde{\Xi}=\left\{\left(\xi_{1}, \omega_{1}\right), \ldots,\left(\xi_{\kappa}, \omega_{\kappa}\right)\right\}$ in $X \times V$ and consider the associated Lagrange basis $\left\{\widetilde{u}_{1}, \ldots, \widetilde{u}_{\kappa}\right\}$ of $\tilde{\mathcal{U}}$, i.e.,

$$
\widetilde{u}_{j}\left(\xi_{i}, \omega_{i}\right)=\delta_{i, j}, \quad 1 \leq i, j \leq \kappa .
$$

Then there are $u_{1}, \ldots, u_{\kappa} \in \mathcal{U}$ such that

$$
\widetilde{u}_{i}(x, v)=\left\langle u_{i}(x), v\right\rangle, \quad x \in X, v \in V,
$$

and $\left\{u_{1}, \ldots, u_{\kappa}\right\}$ forms a basis of $\mathcal{U}$. Consequently, every $u \in \mathcal{U}$ has the representation

$$
u(x)=\sum_{i=1}^{\kappa}\left\langle u\left(\xi_{i}\right), \omega_{i}\right\rangle u_{i}(x), \quad x \in X .
$$

We call $\left\{u_{1} \ldots, u_{\kappa}\right\}$ a Lagrange basis of $\mathcal{U}$ (with respect to the set $\widetilde{\Xi}$ ). 
To proceed with the construction fix a set $\widetilde{\Xi}$ and the resulting Lagrange basis $\left\{u_{1}, \ldots, u_{\kappa}\right\}$ of $\mathcal{U}$. Define a negative definite, hermitian form on $\mathcal{U}$ by setting

$$
\left(u_{i}, u_{j}\right)=-\delta_{i, j}, \quad 1 \leq i, j \leq \kappa .
$$

It remains to determine a Hilbert space $\mathcal{U}^{\perp}$ such that we can define the Pontryagin space $\mathcal{H}$ as the direct sum of $\mathcal{U}$ and $\mathcal{U}^{\perp}$.

From the representation (4.1) the orthogonal projection $f_{\mathcal{U}}$ of a function $f \in \mathcal{H}$ onto $\mathcal{U}$ is given by

$$
f_{\mathcal{U}}=-\sum_{i=1}^{\kappa}\left(f, u_{i}\right) u_{i}=\sum_{i=1}^{\kappa}\left\langle f\left(\xi_{i}\right), \omega_{i}\right\rangle u_{i}
$$

Thus, we can subtract from $\Phi$ any component lying in $\mathcal{U}$ to determine a new kernel $\Psi$. Doing so, we obtain for $x, y \in X$,

$$
\begin{aligned}
\Psi(x, y)=\Phi(x, y)-\sum_{i=1}^{\kappa} \Phi\left(x, \xi_{i}\right) \omega_{i} u_{i}(y)^{*} & -\sum_{i=1}^{\kappa} u_{i}(x) \omega_{i}^{*} \Phi\left(\xi_{i}, y\right) \\
& +\sum_{i, j=1}^{\kappa}\left\langle\Phi\left(\xi_{i}, \xi_{j}\right) \omega_{j}, \omega_{i}\right\rangle u_{i}(x) u_{j}(y)^{*} .
\end{aligned}
$$

By construction we obtain that for all $x \in X$ and $v \in V$,

$$
\left\langle\Psi\left(x, \xi_{r}\right) \omega_{r}, v\right\rangle=0, \quad 1 \leq r \leq \kappa .
$$

Further on, $\Psi(x, y)=\Psi(y, x)^{*}, x, y \in X$.

We are now ready to proceed with the GNS-construction.

Proposition 4.1. Let $V$ be a Pontryagin space and $\Phi: X \times X \rightarrow B(V)$ a conditionally positive definite kernel with respect to the $\kappa$-dimensional space $\mathcal{U}$. Let $\Psi$ be given by (4.2). Then the space

$$
\mathcal{T}=\operatorname{span}\{\Psi(\cdot, y) v: y \in X, v \in V\}
$$

is a pre-Hilbert space and $\mathcal{T} \cap \mathcal{U}=\{0\}$.

Proof. Given the Lagrange basis of $\mathcal{U}$, every element $u$ in $\mathcal{U}$ is uniquely determined by the values $\left\langle u\left(\xi_{r}\right), \omega_{r}\right\rangle, 1 \leq r \leq \kappa$, (cf. (4.10). Thus, the second assertion follows from equation (4.3).

To proof the assertion on the space $\mathcal{T}$ we show that $\Psi$ is positive definite. Then Proposition 3.1 applied to the kernel $\Psi$ yields the assertion.

Towards this end, choose finitely many points $x_{1}, \ldots, x_{n} \in X$ and vectors $v_{1}, \ldots, v_{n} \in V$. The defining equation (4.2) of $\Psi$ allows to obtain that

$$
\begin{aligned}
\sum_{l=1}^{n} \Psi_{x_{l}} v_{l}= & \sum_{l=1}^{n} \Phi_{x_{l}} v_{l}+\sum_{i=1}^{\kappa}\left(-\sum_{l=1}^{n}\left\langle v_{l}, u_{i}\left(x_{l}\right)\right\rangle\right) \Phi_{\xi_{i}} \omega_{i} \\
& +\sum_{i=1}^{\kappa}\left(\sum_{l=1}^{n}\left\langle\sum_{j=1}^{\kappa}\left\langle v_{l}, u_{j}\left(x_{l}\right)\right\rangle \Phi\left(\xi_{i}, \xi_{j}\right) \omega_{j}-\Phi\left(\xi_{i}, x_{l}\right) v_{l}, \omega_{i}\right\rangle\right) u_{i} .
\end{aligned}
$$

Now setting for all $1 \leq r \leq n+\kappa$,

$$
y_{r}= \begin{cases}x_{r}, & 1 \leq r \leq n \\ \xi_{r-n}, & n+1 \leq r \leq n+\kappa,\end{cases}
$$


and

$$
d_{r}= \begin{cases}v_{r}, & 1 \leq r \leq n \\ -\sum_{l=1}^{n}\left\langle v_{l}, u_{r}\left(x_{l}\right)\right\rangle \omega_{r}, & n+1 \leq r \leq n+\kappa\end{cases}
$$

we have that

$$
\sum_{l=1}^{n} \Psi_{x_{l}} v_{l}=\sum_{r=1}^{n+\kappa} \Phi_{y_{r}} d_{r}-\sum_{i=1}^{\kappa}\left\langle\sum_{r=1}^{n+\kappa} \Phi\left(\xi_{i}, y_{r}\right) d_{r}, \omega_{i}\right\rangle u_{i} .
$$

Further, note that

$$
\sum_{r=1}^{n+\kappa}\left\langle u\left(y_{r}\right), d_{r}\right\rangle=0 \quad \forall u \in \mathcal{U}
$$

Therefore, we obtain that

$$
\begin{aligned}
\sum_{k, l=1}^{n}\left\langle\Psi\left(x_{k}, x_{l}\right) v_{l}, v_{k}\right\rangle= & \sum_{k=1}^{n}\left\langle\sum_{r=1}^{n+\kappa} \Phi\left(x_{k}, y_{r}\right) d_{r}, v_{k}\right\rangle \\
& -\sum_{k=1}^{n}\left\langle\sum_{r=1}^{n+\kappa} \sum_{i=1}^{\kappa}\left\langle\Phi\left(\xi_{i}, y_{r}\right) d_{r}, \omega_{i}\right\rangle u_{i}\left(x_{k}\right), v_{k}\right\rangle \\
= & \sum_{r=1}^{n+\kappa} \sum_{s=1}^{n}\left\langle\Phi\left(y_{s}, y_{r}\right) d_{r}, d_{s}\right\rangle \\
& -\sum_{r=1}^{n+\kappa} \sum_{i=1}^{\kappa}\left\langle\Phi\left(\xi_{i}, y_{r}\right) d_{r}, \sum_{k=1}^{n}\left\langle v_{k}, u_{i}\left(x_{k}\right)\right\rangle \omega_{i}\right\rangle \\
= & \sum_{r=1}^{n+\kappa} \sum_{s=1}^{n+\kappa}\left\langle\Phi\left(y_{s}, y_{r}\right) d_{r}, d_{s}\right\rangle \geq 0,
\end{aligned}
$$

since $\Phi$ is conditionally positive definite.

Following the standard GNS-construction we can define $\mathcal{H}_{\Psi}$ to be the Hilbert space completion of $\mathcal{T}$. As in the classical case, $\Psi$ will be the reproducing kernel of $\mathcal{H}_{\Psi}$.

Setting

$$
\mathcal{H}=\mathcal{H}_{\Psi} \oplus \mathcal{U},
$$

endowed with the indefinite inner product

$$
(f, g)=\left(f_{\mathcal{H}_{\Psi}}, g_{\mathcal{H}_{\Psi}}\right)+\left(f_{\mathcal{U}}, g_{\mathcal{U}}\right),
$$

defines a reproducing kernel Pontryagin space of index $\kappa$ with $\mathcal{U}$ being a maximal negative subspace.

Theorem 4.2. Let $\Phi: X \times X \rightarrow B(V)$ be a conditionally positive definite kernel with respect to a $\kappa$-dimensional space $\mathcal{U}$ of $V$-valued functions on $X$. Then there exists a reproducing kernel Pontryagin space $\mathcal{H}$ of index $\kappa$ with maximal negative subspace $\mathcal{U}$. Let $\widetilde{\mathcal{U}}$ be the space of scalar-valued functions in tilde correspondence to $\mathcal{U}$. Choose a $\widetilde{\mathcal{U}}$-unisolvent set $\left\{\left(\xi_{1}, \omega_{1}\right), \ldots,\left(\xi_{\kappa}, \omega_{\kappa}\right)\right\} \subseteq X \times V$ and let $\left\{u_{1}, \ldots, u_{\kappa}\right\}$ 
be the associated Lagrange basis of $\mathcal{U}$. Then the reproducing kernel of $\mathcal{H}$ is given by

$$
\begin{aligned}
\Theta(x, y)=\Phi(x, y)-\sum_{i=1}^{\kappa} & \Phi\left(x, \xi_{i}\right) \omega_{i} u_{i}(y)^{*}-\sum_{i=1}^{\kappa} u_{i}(x) \omega_{i}^{*} \Phi\left(\xi_{i}, y\right) \\
& +\sum_{i, j=1}^{\kappa}\left\langle\Phi\left(\xi_{i}, \xi_{j}\right) \omega_{j}, \omega_{i}\right\rangle u_{i}(x) u_{j}(y)^{*}-\sum_{i=1}^{\kappa} u_{i}(x) u_{i}(y)^{*}
\end{aligned}
$$

for $x, y \in X$.

For the special choice of $\mathcal{U}$ as maximal negative subspace we can state an analog of Theorem 3.4

Corollary 4.3. Let $\Phi: X \times X \rightarrow B(V)$ be a conditionally positive definite kernel with respect to a $\kappa$-dimensional space $\mathcal{U}$ of $V$-valued functions on $X$ and $\mathcal{H}$ as defined above. Then $\mathcal{H}$ endowed with $[\cdot, \cdot]_{\mathcal{U}}$ is a Hilbert space having reproducing kernel

$$
\begin{aligned}
\Lambda(x, y)=\Phi(x, y)-\sum_{i=1}^{\kappa} & \Phi\left(x, \xi_{i}\right) \omega_{i} u_{i}(y)^{*}-\sum_{i=1}^{\kappa} u_{i}(x) \omega_{i}^{*} \Phi\left(\xi_{i}, y\right) \\
& +\sum_{i, j=1}^{\kappa}\left\langle\Phi\left(\xi_{i}, \xi_{j}\right) \omega_{j}, \omega_{i}\right\rangle u_{i}(x) u_{j}(y)^{*}+\sum_{i=1}^{\kappa} u_{i}(x) u_{i}(y)^{*}
\end{aligned}
$$

for $x, y \in X$.

At the end of this section we want to study the dependency of the space $\mathcal{H}$ on the choice of the $\widetilde{\mathcal{U}}$-unisolvent set of points. Doing so, let

$$
\widetilde{\Xi}_{1}=\left\{\left(\xi_{1}^{(1)}, \omega_{1}^{(1)}\right), \ldots,\left(\xi_{\kappa}^{(1)}, \omega_{\kappa}^{(1)}\right)\right\} \text { and } \widetilde{\Xi}_{2}=\left\{\left(\xi_{1}^{(2)}, \omega_{1}^{(2)}\right), \ldots,\left(\xi_{\kappa}^{(2)}, \omega_{\kappa}^{(2)}\right)\right\}
$$

be two $\widetilde{\mathcal{U}}$-unisolvent sets with corresponding Lagrange bases $\left\{u_{1}^{(1)}, \ldots, u_{\kappa}^{(1)}\right\}$ and $\left\{u_{1}^{(2)}, \ldots, u_{\kappa}^{(2)}\right\}$ of $\mathcal{U}$. Denote the associated projected kernels by $\Psi^{(1)}$ and $\Psi^{(2)}$, respectively, and the corresponding reproducing kernel Pontryagin spaces by $\mathcal{H}^{(1)}$ and $\mathcal{H}^{(2)}$, respectively. Starting with

$$
f=\sum_{l=1}^{n} \Psi_{x_{l}}^{(1)} v_{l}
$$

where $x_{1}, \ldots, x_{n} \in X$ and $v_{1}, \ldots, v_{n} \in V$, we show that $f$ has a representation as the sum of an element in $\mathcal{H}_{\Psi^{(2)}}$ and an element of $\mathcal{U}$. Therefore, $f \in \mathcal{H}^{(2)}$. Setting $y_{1}, \ldots, y_{n+\kappa} \in X$ and $d_{1}, \ldots, d_{n+\kappa} \in V$ as in the proof of Proposition 4.1 we can write $f$ in terms of $\Phi$; to be precise,

$$
f=\sum_{l=1}^{n} \Psi_{x_{l}}^{(1)} v_{l}=\sum_{r=1}^{n+\kappa} \Phi_{y_{r}} d_{r}-\sum_{i=1}^{\kappa}\left\langle\sum_{r=1}^{n+\kappa} \Phi\left(\xi_{i}^{(1)}, y_{r}\right) d_{r}, \omega_{i}^{(1)}\right\rangle u_{i}^{(1)} .
$$

On the other hand, we obtain that

$$
\sum_{r=1}^{n+\kappa} \Phi_{y_{r}} d_{r}=\sum_{r=1}^{n+\kappa} \Psi_{y_{r}}^{(2)} d_{r}+\sum_{i=1}^{\kappa}\left\langle\sum_{r=1}^{n+\kappa} \Phi\left(\xi_{i}^{(2)}, y_{r}\right) d_{r}, \omega_{i}^{(2)}\right\rangle u_{i}^{(2)}
$$

Thus, we have shown that $f \in \mathcal{H}^{(2)}$. Denote by $[\cdot, \cdot]_{2}$ the Hilbert space inner product (cf. (2.2) ) with respect to the fundamental decomposition $\mathcal{H}^{(2)}=\mathcal{H}_{\Psi^{(2)}} \oplus \mathcal{U}$. 
Using the fact that

$$
\sum_{r=1}^{n+\kappa}\left\langle u\left(y_{r}\right), d_{r}\right\rangle=0 \quad \forall u \in \mathcal{U}
$$

a straightforward calculation shows that

$$
[f, f]_{2}=(f, f)_{\Psi^{(1)}}+\sum_{r=1}^{\kappa}\left|\left\langle f\left(\xi_{r}^{(2)}\right), \omega_{r}^{(2)}\right\rangle\right|^{2} .
$$

Theorem 4.4. Let $\Phi: X \times X \rightarrow B(V)$ be a conditionally positive definite kernel with respect to a $\kappa$-dimensional space $\mathcal{U}$ of $V$-valued functions on $X$. Let $\widetilde{\mathcal{U}}$ be the space of scalar-valued functions in tilde correspondence to $\mathcal{U}$. Choosing two different $\widetilde{\mathcal{U}}$-unisolvent sets $\widetilde{\Xi}_{1}, \widetilde{\Xi}_{2}$ the resulting reproducing kernel Pontryagin spaces $\mathcal{H}^{(1)}$ and $\mathcal{H}^{(2)}$ coincide as sets and the induced topologies are equivalent.

Proof. We use the same notation as before. Let $g \in \mathcal{H}^{(1)}$. By definition of $\mathcal{H}^{(1)}$ we can write

$$
g=f+u
$$

for some $f \in \mathcal{H}_{\Psi^{(1)}}$ and $u \in \mathcal{U}$. Furthermore, for $f \in \mathcal{H}_{\Psi^{(1)}}$ there exists a sequence $\left(f_{n}\right)_{n \in \mathbb{N}}$ in $\mathcal{T}_{\Psi^{(1)}}$ converging to $f$ in norm. Using the reproducing property of $\Psi^{(1)}$ we obtain

$$
\lim _{n \rightarrow \infty}\left\langle f_{n}(x), v\right\rangle=\langle f(x), v\rangle
$$

for all $x \in X$ and all $v \in V$. By equation (4.4) the sequence $\left(f_{n}\right)_{n \in \mathbb{N}}$ is a Cauchy sequence with respect to $[\cdot, \cdot]_{2}$. Therefore, there exists a limit $h \in \mathcal{H}^{(2)}$. As before,

$$
\langle h(x), v\rangle=\lim _{n \rightarrow \infty}\left\langle f_{n}(x), v\right\rangle=\langle f(x), v\rangle
$$

for all $x \in X$ and all $v \in V$. Since $V$ is non-degenerate this implies $h=f$. Thus, $g=f+u=h+u \in \mathcal{H}^{(2)}$. Finally, exchanging the role of $\mathcal{H}^{(1)}$ and $\mathcal{H}^{(2)}$ yields the assertion.

\section{Applications}

In this section we present two applications of the previously demonstrated constructions. We give a local error estimate for a vector-valued interpolation problem and consider unitary representations of groups in Pontryagin spaces.

Interpolation problem. Let $(V,\langle\cdot, \cdot\rangle)$ be a Pontryagin space and let $X \neq \emptyset$. Assume we are given a finite set $\left\{\left(x_{1}, d_{1}\right), \ldots,\left(x_{n}, d_{n}\right)\right\} \subseteq X \times V$ of samples of an unknown $V$-valued function $f: X \rightarrow V$, i.e.,

$$
f\left(x_{i}\right)=d_{i}, \quad 1 \leq i \leq n .
$$

The goal is to determine a suitable interpolant to the function based on a given strictly conditionally positive definite kernel $\Phi$ with respect to $\mathcal{U}$. Here, strictly conditionally positive definite means that inequality (1.1) holds in the strict sense for mutually different points and non-simultaneously vanishing vectors $v_{1}, \ldots, v_{n} \in$ $V$.

Let $\kappa=\operatorname{dim} \mathcal{U}$ and assume $\left\{x_{1}, \ldots, x_{n}\right\}$ to contain a $\mathcal{U}$-unisolvent set of points. First, let us convince ourselves that we can always find a $\widetilde{\mathcal{U}}$-unisolvent set $\widetilde{\Xi}=$ $\left\{\left(\xi_{1}, \omega_{1}\right), \ldots,\left(\xi_{\kappa}, \omega_{\kappa}\right)\right\}$ such that $\left\{\xi_{1}, \ldots, \xi_{\kappa}\right\} \subseteq\left\{x_{1}, \ldots, x_{n}\right\}$. 
Lemma 5.1. Let $(V,\langle\cdot, \cdot\rangle)$ be a Pontryagin space, $\mathcal{U}$ a $\kappa$-dimensional space of $V$-valued functions on a set $X \neq \emptyset$, and let $\Xi=\left\{x_{1}, \ldots, x_{m}\right\}$ be a $\mathcal{U}$-unisolvent set of points. Let $\widetilde{\mathcal{U}}$ be the space of scalar-valued functions in tilde correspondence to $\mathcal{U}$. Then there exists a finite $\widetilde{\mathcal{U}}$-unisolvent set

$$
\widetilde{\Xi}=\left\{\left(\xi_{1}, \omega_{1}\right), \ldots,\left(\xi_{\kappa}, \omega_{\kappa}\right)\right\} \subseteq X \times V
$$

such that $\left\{\xi_{1}, \ldots, \xi_{\kappa}\right\} \subseteq \Xi$.

Proof. Choose a maximal negative subspace $N$ of $V$ and define

$$
V_{i}=\left\{u\left(x_{i}\right): u \in \mathcal{U}\right\}, \quad 1 \leq i \leq m .
$$

Endowed with the inner product $[\cdot, \cdot]_{N}$ the space $V_{i}$ is a finite-dimensional Hilbert space. For each $V_{i}$ choose an orthonormal basis $\left(v_{j}^{(i)}\right)_{j \in A_{i}}$, where $A_{i} \subseteq\{1, \ldots, \kappa\}$. In the case $V_{i}=\{0\}$ we set $A_{i}=\emptyset$. Then

$$
\widetilde{\Xi}_{0}=\left\{\left(x_{i}, v_{j}^{(i)}\right): j \in A_{i}, 1 \leq i \leq m\right\}
$$

defines a $\widetilde{\mathcal{U}}$-unisolvent set. Indeed, assume there is a function $\widetilde{u} \in \widetilde{\mathcal{U}}$ such that

$$
\widetilde{u}(x, v)=0 \quad \forall(x, v) \in \widetilde{\Xi}_{0} .
$$

Then there exists a function $u \in \mathcal{U}$ such that $\widetilde{u}(x, v)=\langle u(x), v\rangle$ for all $x \in X$ and $v \in V$. From

$$
0=\widetilde{u}\left(x_{i}, v_{j}^{(i)}\right)=\left\langle u\left(x_{i}\right), v_{j}^{(i)}\right\rangle=\left[\left(u\left(x_{i}\right)\right)_{+}-\left(u\left(x_{i}\right)\right)_{-}, v_{j}^{(i)}\right]_{N} \quad \forall j \in A_{i}
$$

we obtain that $\left(u\left(x_{i}\right)\right)_{+}=\left(u\left(x_{i}\right)\right)_{-}$. Therefore, $u\left(x_{i}\right)=0$ for all $1 \leq i \leq m$. Using the $\mathcal{U}$-unisolvency of $\Xi$ we obtain that $u=0$. In turn, this yields $\widetilde{u}=0$. Choose a $\widetilde{\mathcal{U}}$-unisolvent subset $\widetilde{\Xi}$ of $\widetilde{\Xi}_{0}$ that is minimal. It is easy to see that $|\widetilde{\Xi}|=\kappa$.

Let us turn back to the stated interpolation problem. For the given conditionally positive definite kernel $\Phi$ consider the reproducing kernel Pontryagin space $\mathcal{H}$ as in Theorem 4.2. Assume that the unknown function $f$ is contained in $\mathcal{H}$. We make the ansatz

$$
s(x)=\sum_{i=1}^{n} \Phi_{x_{i}} v_{i}+p(x),
$$

where $v_{1}, \ldots, v_{n} \in V$ and $p \in \mathcal{U}$ are determined by

$$
s\left(x_{i}\right)=d_{i}, \quad 1 \leq i \leq n,
$$

and

$$
\sum_{i=1}^{n}\left\langle u\left(x_{i}\right), v_{i}\right\rangle=0 \quad \forall u \in \mathcal{U} .
$$

Choose a $\widetilde{\mathcal{U}}$-unisolvent set $\left\{\left(\xi_{1}, \omega_{1}\right), \ldots,\left(\xi_{\kappa}, \omega_{\kappa}\right)\right\}$ according to Lemma 5.1 and denote by $\Psi$ the projected kernel given in equation (4.2). Choosing $v_{1}, \ldots, v_{n} \in V$ such that

$$
\sum_{i=1}^{n}\left\langle u\left(x_{i}\right), v_{i}\right\rangle=0 \quad \forall u \in \mathcal{U}
$$


we obtain that

$$
\begin{aligned}
\sum_{i=1}^{n} \Phi_{x_{i}} v_{i}= & \sum_{i=1}^{n}\left[\Psi_{x_{i}} v_{i}+\sum_{j=1}^{\kappa}\left\langle v_{i}, u_{j}\left(x_{i}\right)\right\rangle \Phi_{\xi_{j}} \omega_{j}+\sum_{j=1}^{\kappa}\left\langle\Phi\left(\xi_{j}, x_{i}\right) v_{i}, \omega_{j}\right\rangle u_{j}\right. \\
& \left.-\sum_{j, l=1}^{\kappa}\left\langle\Phi\left(\xi_{j}, \xi_{l}\right) \omega_{l}, \omega_{j}\right\rangle\left\langle v_{i}, u_{l}\left(x_{i}\right)\right\rangle u_{j}\right] \\
= & \sum_{i=1}^{n} \Psi_{x_{i}} v_{i}+\sum_{j=1}^{\kappa}\left\langle\sum_{i=1}^{n} \Phi\left(\xi_{j}, x_{i}\right) v_{i}, \omega_{j}\right\rangle u_{j} .
\end{aligned}
$$

Since $f\left(x_{i}\right)=s\left(x_{i}\right), 1 \leq i \leq n, \mathcal{U}$-unisolvency of $\left\{x_{1}, \ldots, x_{n}\right\}$ yields $(f-s)_{\mathcal{U}}=0$. Thus,

$$
\begin{aligned}
{\left[f-s, \sum_{i=1}^{n} \Phi_{x_{i}} v_{i}\right]_{\mathcal{U}} } & =\left(f-s, \sum_{i=1}^{n} \Phi_{x_{i}} v_{i}-\sum_{j=1}^{\kappa}\left\langle\sum_{i=1}^{n} \Phi\left(\xi_{j}, x_{i}\right) v_{i}, \omega_{j}\right\rangle u_{j}\right) \\
& =\sum_{i=1}^{n}\left(f-s, \Psi_{x_{i}} v_{i}\right)=\sum_{i=1}^{n}\left\langle(f-s)\left(x_{i}\right), v_{i}\right\rangle=0 .
\end{aligned}
$$

Therefore, $s$ is the orthogonal projection of $f$ onto

$$
\begin{aligned}
\left\{\sum_{i=1}^{n} \Phi_{x_{i}} w_{i}: \sum_{i=1}^{n}\left\langle u\left(x_{i}\right), w_{i}\right\rangle\right. & =0 \forall u \in \mathcal{U}\}+\mathcal{U} \\
= & \left\{\sum_{i=1}^{n} \Psi_{x_{i}} w_{i}: \sum_{i=1}^{n}\left\langle u\left(x_{i}\right), w_{i}\right\rangle=0 \forall u \in \mathcal{U}\right\} \oplus \mathcal{U} .
\end{aligned}
$$

In particular, $[f-s, s]_{\mathcal{U}}=0$ and, therefore,

$$
\|f\|_{\mathcal{U}}^{2}-\|s\|_{\mathcal{U}}^{2}=\|f-s\|_{\mathcal{U}}^{2} .
$$

If $\mathcal{Q}$ denotes the closure of

$$
\left\{\sum_{i=1}^{n} \Psi_{x_{i}} w_{i}: \sum_{i=1}^{n}\left\langle u\left(x_{i}\right), w_{i}\right\rangle=0 \forall u \in \mathcal{U}\right\}
$$

in $\mathcal{H}_{\Psi}$ we can obtain a bound for the pointwise error (cf. [5, Chapter 31]).

Theorem 5.2. Let $(V,\langle\cdot, \cdot\rangle)$ be a Pontryagin space and $\Phi: X \times X \rightarrow B(V)$ be conditionally positive definite with respect to a finite-dimensional space $\mathcal{U}$. Let $\left\{x_{1}, \ldots, x_{n}\right\} \subseteq X$ be a set of points containing a $\mathcal{U}$-unisolvent set and let $d_{i}=f\left(x_{i}\right)$, $1 \leq i \leq n$, for a function $f \in \mathcal{H}$. For the interpolant $s \in \mathcal{H}$ defined in equation (5.1) we have that

$$
|\langle f(x)-s(x), v\rangle| \leq\|f\|_{\mathcal{U}}\|v\|_{V}^{1 / 2}\left\|\Psi(x, x) v-\left(\operatorname{proj}_{\mathcal{Q}}\left(\Psi_{x} v\right)\right)(x)\right\|_{V}^{1 / 2}
$$

for every $x \in X$ and $v \in V$. Hereby, $\|\cdot\|_{\mathcal{U}}$ denotes the norm associated with the fundamental decomposition $\mathcal{H}=\mathcal{H}_{\Psi} \oplus \mathcal{U}$ and $\|\cdot\|_{V}$ any norm associated with a fundamental decomposition of $V$.

Proof. We already noted that $f-s \in \mathcal{H}_{\Psi}$. Thus,

$$
\begin{aligned}
|\langle f(x)-s(x), v\rangle| & =\left|\left(f-s, \Psi_{x} v\right)\right|=\left|\left(f-s, \Psi_{x} v-\operatorname{proj}_{\mathcal{Q}}\left(\Psi_{x} v\right)\right)\right| \\
& \leq\|f-s\|\left\|\Psi_{x} v-\operatorname{proj}_{\mathcal{Q}}\left(\Psi_{x} v\right)\right\|,
\end{aligned}
$$


where $\|\cdot\|$ denotes the norm in the Hilbert space $\mathcal{H}_{\Psi}$. Using the reproducing property of $\Psi$ we obtain that

$$
\begin{aligned}
\left\|\Psi_{x} v-\operatorname{proj}_{\mathcal{Q}}\left(\Psi_{x} v\right)\right\|^{2} & =\left(\Psi_{x} v-\operatorname{proj}_{\mathcal{Q}}\left(\Psi_{x} v\right), \Psi_{x} v\right) \\
& =\left\langle\Psi(x, x) v-\left(\operatorname{proj}_{\mathcal{Q}}\left(\Psi_{x} v\right)\right)(x), v\right\rangle \\
& \leq\left\|\Psi(x, x) v-\left(\operatorname{proj}_{\mathcal{Q}}\left(\Psi_{x} v\right)\right)(x)\right\|_{V}\|v\|_{V} .
\end{aligned}
$$

Since $\|f-s\|_{\mathcal{U}}=\|f-s\|$, the statement follows from $\|f-s\|_{\mathcal{U}} \leq\|f\|_{\mathcal{U}}$.

Since the projection onto $\mathcal{Q}$ is difficult to calculate we choose an easier accessible element of $\mathcal{Q}$ to obtain an upper bound for the pointwise error in the last theorem.

Corollary 5.3. Adopt the setting of Theorem 5.2, Then

$$
|\langle f(x)-s(x), v\rangle| \leq\|f\|_{\mathcal{U}}\|v\|_{V} \sqrt{\|\Psi(x, x)\|}
$$

for every $x \in X$ and $v \in V$, where $\|\Psi(x, x)\|$ denotes the operator norm.

Proof. Since $\operatorname{proj}_{\mathcal{Q}}\left(\Psi_{x} v\right)$ is the best approximation to $\Psi_{x} v$ in $\mathcal{Q}$ we have for every element $q \in \mathcal{Q}$,

Choosing $q=0$ yields

$$
\left\|\Psi_{x} v-\operatorname{proj}_{\mathcal{Q}}\left(\Psi_{x} v\right)\right\| \leq\left\|\Psi_{x} v-q\right\| .
$$

$$
\left\|\Psi_{x} v-\operatorname{proj}_{\mathcal{Q}}\left(\Psi_{x} v\right)\right\|^{2} \leq\left\|\Psi_{x} v\right\|^{2}=\langle\Psi(x, x) v, v\rangle \leq\|\Psi(x, x)\|\|v\|_{V}^{2} .
$$

Example A. Duchon's work (see, e.g., [18]) on minimal norm interpolation in Sobolev spaces has been generalized for vector-valued functions by several authors (cf., e.g., [2, 7, 10, and the references given therein). These authors used an approach via distributions to define the appropriate reproducing kernel spaces of functions $f: \mathbb{R}^{d} \rightarrow \mathbb{R}^{d}$. Let $\mathcal{D}^{\prime}\left(\mathbb{R}^{d}\right)$ denote the space of distributions in $\mathbb{R}^{d}$. The Beppo-Levi space of order $m \in \mathbb{N}_{0}$ is then defined as

$$
D^{-m} L^{2}\left(\mathbb{R}^{d}\right)=\left\{v \in \mathcal{D}^{\prime}\left(\mathbb{R}^{d}\right): D^{\alpha} v \in L^{2}\left(\mathbb{R}^{d}\right),|\alpha|=m\right\} .
$$

For a $d$-component vector field one then defines

$$
D^{-m} L^{2}\left(\mathbb{R}^{d}, \mathbb{R}^{d}\right)=D^{-m} L^{2}\left(\mathbb{R}^{d}\right) \times \cdots \times D^{-m} L^{2}\left(\mathbb{R}^{d}\right) .
$$

The function

$$
\phi_{0}(x)=\left\{\begin{array}{ll}
\|x\|^{2 m} \log \|x\|, & \text { for } m \text { even, } \\
(-1)^{m}\|x\|^{2 m-1}, & \text { for } m \text { odd, }
\end{array} \quad x \in \mathbb{R}^{d},\right.
$$

is the fundamental solution of the equation $\Delta^{m+1} \phi_{0}=\delta$. Setting $\phi(x, y)=\phi_{0}(x-y)$ we obtain a translation invariant, conditionally positive definite kernel with respect to the space of polynomials $\mathcal{P}_{d}^{m-1}$ in $d$ variables and of total degree at most $m-1$.

Amodei and Benbourhim (for $m=2$ ), and Dodu and Rabut (for $m=3$ ) then considered a minimal norm interpolation problem in the Beppo-Levi space $D^{-m} L^{2}\left(\mathbb{R}^{d}, \mathbb{R}^{d}\right)$ with a semi-norm composed of a rotational and a divergence component, and a balancing parameter $\lambda \geq 0$. This leads to a decomposition of the interpolant according to the two semi-norm components. The first one is determined by the kernel

$$
\Phi_{0}^{r o t}=(-1)^{m}\left[\partial_{i j}^{2}\right]_{i, j=1}^{d} \phi_{0},
$$

while the second one can be represented using the kernel

$$
\Phi_{0}^{\text {div }}=(-1)^{m}\left(\Delta \phi_{0} \cdot I_{d}-\Phi_{0}^{r o t}\right) .
$$


The reproducing kernel of the combined semi-norm in the Beppo-Levi space is finally given by

$$
\Phi_{0}=\Phi_{0}^{d i v}+\frac{1}{\lambda} \Phi_{0}^{r o t}
$$

In a similar way Handscomb 10 studied thin-plate spline techniques for vector fields.

Example B. A related approach has been suggested by Narcowich and Ward in 1994 and later been extended by Lowitzsch and Fuselier (cf. 9 and the references therein). The idea is to derive matrix-valued radial basis functions on $\mathbb{R}^{d}$ by applying differential operators to scalar-valued radial functions. Depending on the differential operator chosen, the authors obtained divergence- and curl-free kernels, respectively. Again, dealing with kernels on $\mathbb{R}^{d}$ one can consider translation invariant kernels and therefore apply Fourier techniques.

Given a translation invariant, scalar-valued kernel $\phi_{0} \in C^{2}\left(\mathbb{R}^{d}\right)$ such that $\Delta \phi_{0} \in$ $L^{1}\left(\mathbb{R}^{d}\right)$, the divergence-free, matrix-valued kernel

$$
\Phi_{0}^{d i v}(x)=\left(-\Delta I+\nabla \nabla^{t}\right) \phi_{0}(x), \quad x \in \mathbb{R}^{d},
$$

generates the reproducing kernel space

$$
\begin{aligned}
\mathcal{H}^{\text {div }}=\left\{f \in L^{2}\left(\mathbb{R}^{d}\right) \cap C\left(\mathbb{R}^{d}\right):\right. \\
\left.\qquad \int_{\mathbb{R}^{d}} \widehat{f}(\xi)^{*} \widehat{\Phi_{0}^{\text {div }}}(\xi)^{+} \widehat{f}(\xi) d \xi<\infty \text { and } e_{\xi}^{t} \widehat{f}(\xi)=0 \text { a.e. }\right\},
\end{aligned}
$$

where $\widehat{\Phi_{0}^{d i v}}(\xi)^{+}$denotes the Moore-Penrose inverse of the matrix $\widehat{\Phi_{0}^{d i v}}(\xi)$, and $e_{\xi}$ is a unit vector in direction $\xi \in \mathbb{R}^{d}$.

If the scalar-valued kernel $\phi_{0}$ in (5.2) satisfies the decay conditions

$$
c_{1}\left(1+\|\xi\|_{2}^{2}\right)^{-(\tau+1)} \leq \widehat{\phi}(\xi) \leq c_{2}\left(1+\|\xi\|_{2}^{2}\right)^{-(\tau+1)}
$$

for some constants $c_{1}, c_{2}$ and $\tau>d / 2$, then the space $\mathcal{H}^{\text {div }}$ can be identified with some Sobolev space of order $\tau$.

Analogously, Fuselier [9] considered a curl-free kernel

$$
\Phi_{0}^{\text {curl }}(x)=-\nabla \nabla^{t} \phi_{0}(x),
$$

the associated reproducing kernel space of which can again be identified as weighted $L^{2}$-space with weight function given by the inverse of the Fourier transform of the kernel $\Phi_{0}^{\text {curl }}$. Again, posing some additional decay conditions, the space can be identified with an appropriate Sobolev space.

Group representation. Consider $X$ to be a group and let $(V,\langle\cdot, \cdot\rangle)$ be a Pontryagin space of index $\gamma$. Let $e \in X$ denote the neutral element in $X$ and $U(V)$ the space of all unitary operators on $V$ with respect to $\langle\cdot, \cdot\rangle$. A map

$$
\pi: X \rightarrow U(V)
$$

satisfying

$$
\pi(x y)=\pi(x) \pi(y), \pi\left(x^{-1}\right)=\pi(x)^{-1}=\pi(x)^{*}, \quad x, y \in X,
$$

is called unitary representation of $X$ in $V$ (cf., e.g., [17).

Choose a maximal negative subspace $N$ of $V$ and let

$$
\mathcal{U}=\left\{\pi(\cdot)^{*} v: v \in N\right\} .
$$


Then

$$
\Phi(x, y)=\pi\left(x^{-1} y\right)
$$

defines a conditionally positive definite kernel $\Phi$ of order $\gamma$ on $X$. To see this, select finitely many points $x_{1}, \ldots, x_{n} \in X$ and vectors $v_{1}, \ldots, v_{n} \in V$ such that

$$
\sum_{i=1}^{n}\left\langle u\left(x_{i}\right), v_{i}\right\rangle=0 \quad \forall u \in \mathcal{U} .
$$

We then obtain that

$$
0=\sum_{i=1}^{n}\left\langle u\left(x_{i}\right), v_{i}\right\rangle=\sum_{i=1}^{n}\left\langle\pi\left(x_{i}\right)^{*} v, v_{i}\right\rangle=\left\langle v, \sum_{i=1}^{n} \pi\left(x_{i}\right) v_{i}\right\rangle
$$

for all $v \in N$. Thus, $\sum_{i=1}^{n} \pi\left(x_{i}\right) v_{i} \in N^{\perp}$ and we can conclude

$$
\begin{aligned}
\sum_{i, j=1}^{n}\left\langle\Phi\left(x_{i}, x_{j}\right) v_{j}, v_{i}\right\rangle & =\sum_{i, j=1}^{n}\left\langle\pi\left(x_{i}\right)^{*} \pi\left(x_{j}\right) v_{j}, v_{i}\right\rangle \\
& =\left\langle\sum_{j=1}^{n} \pi\left(x_{j}\right) v_{j}, \sum_{i=1}^{n} \pi\left(x_{i}\right) v_{i}\right\rangle \geq 0 .
\end{aligned}
$$

We say that $\pi: X \rightarrow U(V)$ is a conditionally positive definite function on $X$ with respect to $\mathcal{U}$. The following theorem sums up our findings concerning group representations in Pontryagin spaces.

Theorem 5.4. Let $X$ be a group and $(V,\langle\cdot, \cdot\rangle)$ be a Pontryagin space of index $\gamma \in \mathbb{N}_{0}$. Any unitary representation $\pi$ of $X$ in $V$ is a conditionally positive definite function on $X$ with respect to a space $\mathcal{U}$ isomorphic to a maximal negative subspace of $V$.

Note that in this case the two constructions result in the same reproducing kernel space, which is isometrically isomorphic to $V$.

\section{REFERENCES}

[1] D. Alpay, A. Dijksma, J. Rovnyak, and H. de Snoo, Schur functions, operator colligations, and reproducing kernel Pontryagin spaces, Operator Theory: Advances and Applications, vol. 96, Birkhäuser, Basel, 1997. MR.1465432 (2000a:47024)

[2] L. Amodei and M. N. Benbourhim, A vector spline approximation, J. Approx. Theory 67 (1991), no. 1, 51-79. MR.1127820 (92i:41012)

[3] G. Berschneider and W. zu Castell, Conditionally positive definite kernels and Pontryagin spaces, Approximation Theory XII. Proceedings of the 12th International Conference, San Antonio, TX, USA, March 4-8, 2007 (M. Neamtu and L. L. Schumaker, eds.), Modern Methods in Mathematics, Nashboro Press, Brentwood, TN, 2008, pp. 27-37. MR.2537117 (2010j:41001)

[4] J. Bognár, Indefinite inner product spaces, Ergebnisse der Mathematik und ihrer Grenzgebiete, vol. 78, Springer, Berlin-Heidelberg-New York, 1974. MR 0467261 (57:7125)

[5] W. Cheney and W. Light, A course in approximation theory, Brooks/Cole, Pacific Grove, CA, 1999.

[6] J.-P. Chilès and P. Delfiner, Geostatistics. Modeling spatial uncertainty, Wiley Series in Probability and Statistics, John Wiley and Sons, New York, NY, 1999. MR 1679557 (2000f:86010)

[7] F. Dodu and C. Rabut, Vectorial interpolation using radial-basis-like functions, Comput. Math. Appl. 43 (2002), no. 3-5, 393-411. MR.1883575 (2003a:65010)

[8] T. Evgeniou, C. A. Micchelli, and M. Pontil, Learning multiple tasks with kernel methods, J. Mach. Learn. Res. 6 (2005), 615-637. MR2249833 
[9] E. J. Fuselier, Improved stability estimates and a characterization of the native space for matrix-valued RBFs, Adv. Comput. Math. 29 (2008), no. 3, 311-313. MR2438347 (2009k:41003)

[10] D. Handscomb, Local recovery of a solenoidal vector field by an extension of the thin-plate spline technique, Numer. Algorithms 5 (1993), no. 1-4, 121-129. MR.1258589

[11] W. Light and H. Wayne, Spaces of distributions, interpolation by translates of a basis function and error estimates., Numer. Math. 81 (1999), no. 3, 415-450. MR1668091 (99m:65021)

[12] G. B. Pedrick, Theory of reproducing kernel Hilbert spaces of vector-valued functions, Studies in Eigenvalue Problems 19, University of Kansas, Department of Mathematics, 1957.

[13] Z. Sasvári, Positive definite and definitizable functions, Mathematical Topics, vol. 2, Akademie Verlag, Berlin, 1994. MR1270018 (95c:43005)

[14] R. Schaback, A unified theory of radial basis functions. Native Hilbert spaces for radial basis functions II, J. Comput. Appl. Math. 121 (2000), no. 1-2, 165-177. MR:1752527 (2002h:41037)

[15] B. Schölkopf and A. J. Smola, Learning with kernels - support vector machines, regularization, optimization, and beyond, MIT Press, Cambridge, MA, 2002.

[16] L. Schwartz, Sous-espaces hilbertiens d'espaces vectoriels topologiques et noyaux associés. (Noyaux reproduisants), J. Anal. Math. 13 (1964), 115-256 (French). MR0179587(31:3835)

[17] P. Sorjonen, Pontrjaginräume mit einem reproduzierenden Kern, Ann. Acad. Sci. Fenn., Ser. I. A. Math. 594 (1975) (German). MR0405079 (53:8875)

[18] H. Wendland, Scattered data approximation, Cambridge Monographs on Applied and Computational Mathematics, vol. 17, Cambridge University Press, Cambridge, 2005. MR 2131724 (2006i:41002)

Institute for Mathematical Stochastics, Technische Universität Dresden, 01062 Dresden, Germany

E-mail address: georg.berschneider@tu-dresden.de

Department of Scientific Computing, Helmholtz Zentrum München, German Research Center for Environmental Health, Ingolstaedter Landstrasse 1, 85764 Neuherberg, Germany

E-mail address: castell@helmholtz-muenchen.de

Department of Scientific Computing, Helmholtz Zentrum München, German Research Center for Environmental Health, Ingolstaedter Landstrasse 1, 85764 Neuherberg, Germany

E-mail address: stefan.schroedl@gmx.net 